\title{
ISOMETRIES OF THE DISC ALGEBRA
}

\author{
MOHAMAD EL-GEBEILY AND JOHN WOLFE
}

\begin{abstract}
The linear isometries $u: A \rightarrow A$ of the disc algebra $A$ into itself are completely described. Such isometries $u$ must be one of two distinct types. The first type is $u f=\psi \cdot f(\phi)$, where $\psi \in A$ and $\phi \in H^{\infty}$ satisfy certain described conditions. The second type is $u f=E(\psi \cdot f(\phi))$, where $\phi: Q \rightarrow T$ is any continuous function from a closed zero measure subset $Q$ of the unit circle $T$ onto itself, $\psi \in C(Q)$ is unimodular, and $E: Y \rightarrow A$ is a norm 1 extension operator, where $Y=\{\psi \cdot f(\phi): f \in A\} \subset C(Q)$. Isometries of $C(K)$ spaces into the disc algebra are also described.
\end{abstract}

1. Introduction. A linear operator $u: X \rightarrow X$ on a Banach space $X$ is an isometry if $\|u x\|=\|x\|$ for each $x \in X$. The isometries of most of the well-known Banach spaces have been described. The isometries of $C(K)$ spaces were described by Banach and Stone (onto case) and Holsztynski [12] (into case). Isometries of $L^{p}(\mu)$ spaces and $H^{p}$ were worked out by Lamperti [16] and Forelli [9], respectively. The onto isometries of the disc algebra $A$ and $H^{\infty}$ were determined by de Leeuw, Rudin, and Wermer [17]. Several further papers dealing with isometries on various spaces are listed in the bibliography. In this paper we describe the isometries of the disc algebra $A$ into itself. This answers a question raised by Phelps [30, p. 354].

Our notation follows Rudin [26] and Hoffman [11]. We use $D$ for the open unit disc in the complex plane, $\bar{D}=\{z:|z| \leq 1\}$, and $T=\{z:|z|=1\}$. We use $C(T)$, $C(\bar{D})$, and $C(K)$ to denote the sup norm Banach spaces of continuous complex valued functions on $T, \bar{D}$, or a general compact Hausdorff space $K$, respectively. The disc algebra $A=\{f \in C(\bar{D}): f$ is analytic on $D\}$, and $H^{\infty}$ is the sup norm Banach space of bounded analytic functions on $D$. Lebesgue measure on $T$ is denoted by $m$. We identify $A$ in its natural way as a subspace of $C(T)$.

We begin by discussing Propositions 1 and 2 which describe two types of isometries of the disc algebra into itself. The main result (Theorem A) is that any isometry on $A$ must be of the form described in either Propositions 1 or 2 . Theorem $\mathrm{B}$ describes the isometries of $C(K)$ spaces into $A$.

These results are proved in $\S 2$. $\S 3$ contains a few further remarks and open questions.

Proposition 1. Suppose $\phi \in H^{\infty}$ and $\|\phi\| \leq 1$, where $\phi=h_{1} / h_{2}$ with $h_{1}, h_{2} \in$ $A$. Let $S=\left\{t \in T: h_{2}(t)=0\right\}$. Suppose $\psi \in A$ and $\psi(s)=0$ for $s \in S$.

(a) Then $u f=\psi \cdot f(\phi)$ defines a bounded linear operator from $A$ into $A$.

(b) The operator $u$ is an isometry $\Leftrightarrow\|\phi\|=\|\psi\|=1$, and there is a closed set $Q$ in $T$ such that $Q \cap S=\varnothing, \phi(Q)=T$ and $|\psi(q)|=1$ for all $q \in Q$.

An isometry $u: A \rightarrow A$ of the form described in Proposition 1(b) will be called a Type 1 isometry on $A$.

Received by the editors December 12, 1983 and, in revised form, May 9, 1984.

1980 Mathematics Subject Classification. Primary 30H05, 46E25.

(C) 1985 American Mathematical Society $0002-9939 / 85 \$ 1.00+\$ .25$ per page 
McDonald [33, Proposition 1.1] shows that isometries as in part (b) are precisely the ones satisfying $(u 1) u(f g)=(u f)(u g)$.

Type 1 isometries are quite natural and expected in this situation, since for many function spaces $X$ (e.g., on $H^{p}$ or $L^{p}$ ) all isometries are of the form $u f=\psi \cdot f(\phi)$, where the conditions on $\psi$ and $\phi$ depend on the nature of $X$.

A wide variety of allowable functions $\phi$ and $\psi$, for which $u f=\psi \cdot f(\phi)$ is an isometry of $A$, can be imagined. For example, if $\phi_{1} \in A$ is a "Riemann map" of $\bar{D}$ to the quarter annulus $\{z: 1 / 2 \leq z \leq 1$ and $0 \leq \arg z \leq \pi / 2\}$, then some proper arc $Q$ of $T$ is mapped onto $\{z:|z|=1$ and $0<\arg z<\pi / 2\}$. Thus $u f=\psi f(\phi)$ is an isometry, where $\phi=\left(\phi_{1}\right)^{4}$ and $\psi \in A$ is any norm 1 function which is unimodular on $Q$.

We also note that $\phi \in H^{\infty}$ is of the form $\phi=h_{1} / h_{2}$, where $h_{1}, h_{2} \in A \Leftrightarrow$ the radial limit of $\phi$ is continuous off some closed subset of $T$ with measure zero.

The next proposition is trivial.

Proposition 2. Let $Q$ be a closed subset of $T$ of measure zero, let $\phi: Q \rightarrow T$ be any continuous onto map, and let $\psi \in C(Q)$ satisfy $|\psi(q)|=1$ for each $q \in Q$. Define the subspace $Y$ of $C(Q)$ by $Y=\{\psi \cdot f(\phi): f \in A\}$. Let $E: Y \rightarrow A$ be a linear extension operator with $\|E\|=1$, i.e., $E$ is a bounded linear operator of norm 1 such that, for each $f \in Y$ and $q \in Q, E(f)(q)=f(q)$. Then $u f=E(\psi \cdot f(\phi))$ defines an isometry of $A$ into $A$.

An isometry $u: A \rightarrow A$ of the form described in Proposition 2 will be called a Type 2 isometry on $A$.

We are now in a position to state our main result.

THEOREM A. Any isometry of $A$ is either of Type 1 or of Type 2, i.e., of the form described in either Proposition 1 or Proposition 2.

One reason for the existence of what we have called Type 2 isometries on $A$ is that $A$ contains isometric copies of $C(T)$ (see Pelczynski [21]). This second type of isometry previously appeared (in the context of Banach spaces of the type $C(K) . K$ a compact Hausdorff space) in Holsztynski [12] (see also Proposition 1 of Pelczynski $[\mathbf{2 3}])$.

In order to illuminate the nature of Type 2 isometries, we make the following observations.

We note that for any closed subset $Q$ of $T$ of measure zero there are many norm 1 extension operators $E: C(Q) \rightarrow A$ as shown by Pelczynski [21] and Michael and Pelczynski [18] (also see Rudin [25], Carleson [6], and Bishop [4]).

We also note that if $Q \subset T$ has measure zero then there exist continuous maps $\phi: Q \rightarrow T$ of $Q$ onto $T \Leftrightarrow Q$ is uncountable (e.g., $Q$ is a homeomorph of the Cantor set). This argument goes as follows: $(1) m(Q)=0$ implies $Q$ is totally disconnected (it cannot contain intervals); (2) $Q$ uncountable implies that it contains a homeomorph of the Cantor set which must be a retract of $Q ;(3)$ there are well-known maps of the Cantor set onto $T$.

Thus we see that Type 2 isometries exist in profusion. Unfortunately, it seems that Type 2 isometries can never be described very explicitly, since, firstly, the maps $\phi: Q \rightarrow T$, in the few cases in which they are explicit, are rather ugly, and, secondly, the extension operators $E: C(Q) \rightarrow A$, which are constructed by Michael and Pelczynski using a limiting process, always seem to be illusive. 
Our final result describes the isometries of a $C(K)$ space into $A$.

THEOREM B. Let $K$ be a compact metric space, and let $u: C(K) \rightarrow A$ be an isometry. Then $u$ is Type 2. More precisely, $u f=E(\psi \cdot f(\phi))$, where $Q$ is a closed subset of $T$ of measure zero, $\phi: Q \rightarrow K$ is continuous and onto, $\psi \in C(Q)$ satisfies $\psi(q)=1$ for all $q \in Q$, and $E: Y \rightarrow A$ is a norm 1 extension operator, where $Y=\{\psi \cdot f(\phi): f \in C(K)\}$.

2. Proof of the results. Our basic tool for proving Theorems A and B is the following proposition.

Proposition 3. Let $u: A \rightarrow A$ be an isometry. Then there exist a closed subset $Q$ of $T$, a continuous map $\rho: Q \rightarrow T$, and a continuous onto map $\phi: Q \rightarrow T$ such that $\rho(q) u(g)(q)=g(\phi(q))$ for all $g \in A$ and $q \in Q$.

We identify $A^{*}$ as a quotient space of $M(T)$, where $M(T)=C(T)^{*}$ denotes the space of regular Borel measures on $T$. The notation $B\left(A^{*}\right), B\left(C(T)^{*}\right)$, and ext $B\left(A^{*}\right)$ and ext $B\left(C(T)^{*}\right)$ denote, respectively, the unit balls and extreme points of the unit balls of $A^{*}$ and $C(T)^{*}=M(T)$. For $t \in T, \delta_{t} \in A^{*}$ denotes the point evaluation. Since $t \rightarrow \delta_{t}$ is a homeomorphism of $T$ into $A^{*}$ equipped with the weak* topology, we identify $T$ with the subset $\left\{\delta_{t}: t \in T\right\}$ of $A^{*}$.

The following proof is a straightforward adaptation of Pelczynski's proof $[\mathbf{2 3}$, Proposition 1] of a result of Holsztynski [12] on isometries of $C(K)$ spaces.

PROOF OF PROPOSITION 3. We first establish that for each $t \in T$ the set $K_{t} \neq$ $\varnothing$, where $K_{t}=\left(\left(u^{*}\right)^{-1} \delta_{t}\right) \cap \operatorname{ext} B\left(A^{*}\right)$. First of all, $\tilde{K}_{t}=\left(\left(u^{*}\right)^{-1} \delta_{t}\right) \cap B\left(A^{*}\right) \neq \varnothing$ because $u$ is an isometry and, thus, $u^{*}\left(B\left(A^{*}\right)\right)=B\left(A^{*}\right)$. But $\tilde{K}_{t}$ (using the terminology of $\S \mathrm{V} .8$ of Dunford and Schwartz [7]) is a weak* compact extremal subset of $B\left(A^{*}\right)$, so it has extreme points which will also be extreme points of $B\left(A^{*}\right)$, which shows that $K_{t} \neq \varnothing$.

Now for each $\lambda \in T$ let $Q_{\lambda}=\left(u^{*-1}(\lambda T)\right) \cap T$ and let $Q=\bigcup_{\lambda \in T} Q_{\lambda}$. Define $\rho: Q \rightarrow T$ by $\rho(q)=\lambda^{-1}$ if $q \in Q_{\lambda}$, and define $\phi: Q \rightarrow T$ by $\phi(q)=\rho(q) u^{*}\left(\delta_{q}\right)$. The last paragraph shows that $\phi$ maps $Q$ onto $T$ (since $\operatorname{ext} B\left(A^{*}\right)=\left\{\alpha \delta_{t}: t \in\right.$ $T,|\alpha|=1\})$. Also, by definition, for $q \in Q$ and $g \in A$,

$$
\rho(q)(u g)(q)=\rho(q)\left(u^{*}\left(\delta_{q}\right)\right) g=\delta_{\phi(q)} g=g(\phi(q)) .
$$

To see that $Q$ is closed and $\rho$ is continuous, let $F$ be a closed subset of $T$. Then

$$
\begin{aligned}
\rho^{-1}(F) & =\bigcup_{\lambda \in F} Q_{\lambda-1}=\bigcup_{\lambda \in F}\left(u^{*-1}\left(\lambda^{-1} T\right) \cap T\right) \\
& =\left(u^{*-1}\left(\bigcup_{\lambda \in F} \lambda^{-1} T\right)\right) \cap T=\left(u^{*-1}\left(F^{-1} \times T\right)\right) \cap T
\end{aligned}
$$

is weak ${ }^{*}$ closed since

$$
F^{-1} \times T=\left\{\lambda^{-1} t: \lambda \in F, t \in T\right\}
$$

is closed and $u^{*}$ is weak ${ }^{*}$ continuous. Then $\rho$ is continuous and $Q=\rho^{-1}(T)$ is closed. This proves Proposition 3.

Proof of Proposition 1. To see $u f=\psi \cdot f(\phi)$ is bounded from $A$ to $A$ observe that, for $f \in A, u f$ is analytic on $D$ and, for $z \in D,|u f(z)| \leq\|\psi\|\|f\|$, so $u$ is bounded from $A$ into $H^{\infty}$. To get $u(A) \subset A$ we need $u\left(z^{n}\right) \in A$ for each $n \geq 1$. 
But $u z^{n}=\psi h_{1}^{n} / h_{2}^{n}$, and for $t \in T \backslash S$ this is certainly continuous. If $t_{0} \in S$ then

$$
\lim _{t \rightarrow t_{0}}\left|\left(u z^{n}\right)(t)\right| \leq \lim _{t \rightarrow t_{0}}|\psi(t)| \varlimsup_{t \rightarrow t_{0}}\left|\phi^{n}(t)\right|=0,
$$

since $\phi \in H^{\infty}$ and $\psi\left(t_{0}\right)=0$. This proves (a).

For (b) we first note that, since $Q \cap S=\varnothing$, although $\phi \in H^{\infty}$, its radial limit is continuous on $T-S$ so that $\phi(Q)$ makes sense. The proof that a Type 1 operator is an isometry is straightforward. Conversely, if $u f=\psi \cdot f(\phi)$ is an isometry, then the set $Q$ is obtained from Proposition 3. Taking $g$ in Proposition 3 to be 1 gives $|\psi(q)|=\left|\rho(q)^{-1}\right|=1$ for $q \in Q$ (hence, $Q \cap S$ is void). Taking $g$ to be $z$ gives the desired conclusion $\phi(Q)=T$, and the rest of the proof is apparent.

Proof of TheOrem A. Given the isometry $u: A \rightarrow A$ let $Q, \rho: Q \rightarrow T$ and $\phi: Q \rightarrow T$ be as given in Proposition 3, i.e., for $q \in Q, \rho(q)(u f)(q)=f(\phi(q))$. We will show that if $m(Q)>0$ then $u$ is a Type 1 isometry, and if $m(Q)=0$ then $u$ is a Type 2 isometry.

So first assume $m(Q)>0$. The proof of this case is similar to the proof of Theorem 1.1 of McDonald [33].

Letting $f=1$ we get, for $q \in Q, \rho(q)(u 1) q=1$ or $u 1=1 / \rho$ on $Q$. Thus,

$$
u f(q)=(u 1)(q) f(\phi(q)) \text { for } f \in A \text { and } q \in Q .
$$

Next we establish

$$
(u 1)(u(f g))=(u f)(u g) \text { for } f, g \in A .
$$

For from (1), for $q \in Q$,

$$
(u 1)(q) u(f g)(q)=(u 1(q))^{2} f(\phi(q)) g(\phi(q))=(u f)(q)(u g)(q) .
$$

Thus, (2) holds on $Q$. But $m(Q)>0$ and the functions involved are in $A$, so (2) holds on $\bar{D}$.

It follows immediately from (2) that

$$
(u 1)^{n-1} u\left(z^{n}\right)=(u z)^{n} \quad \text { for } n \geq 1 .
$$

Now define $\phi_{1}(\xi)=u z(\xi) / u 1(\xi)$. We now show that $\phi_{1}$ is analytic on $D$ and $\left\|\phi_{1}\right\|=1$.

Suppose $u 1$ has a zero of order $n \geq 1$ at $\xi_{0} \in D$, i.e., $\lim _{\xi \rightarrow \xi_{0}}\left[(u 1)(\xi) /\left(\xi-\xi_{0}\right)^{n}\right]$ exits. So by (3),

$$
\left[u(z)(\xi) /\left(\xi-\xi_{0}\right)^{n-1}\right]^{n}=\left[u 1(\xi) /\left(\xi-\xi_{0}\right)^{n}\right]^{n-1} u\left(z^{n}\right)(\xi) .
$$

But $u\left(z^{n}\right)\left(\xi_{0}\right)=0$ since, by $(2)$,

$$
0=(u 1)\left(\xi_{0}\right) u\left(z^{2 n}\right)\left(\xi_{0}\right)=\left[\left(u z^{n}\right)\left(\xi_{0}\right)\right]^{2} .
$$

Thus $\lim _{\xi \rightarrow \xi_{0}}\left[u(z)(\xi) /\left(\xi-\xi_{0}\right)^{n-1}\right]=0$, and $u z$ has a zero of order at least $n$ at $\xi_{0}$, so $\phi_{1}$ is analytic on $D$.

To get $\left\|\phi_{1}\right\| \leq 1$ note that, by $(3),\left(u z^{n}\right)=(u 1)(\xi) \phi_{1}^{n}(\xi)$. Thus, if $u 1(\xi) \neq 0$, then

$$
\left|\phi_{1}(\xi)\right|^{n} \leq\left\|u z^{n}\right\| /|u 1(\xi)| \leq 1 /|u 1(\xi)| \text { for all } n \text {. }
$$

Thus, $\left|\phi_{1}(\xi)\right| \leq 1$. Since the zeros of the $u 1$ are isolated, we get $\left\|\phi_{1}\right\| \leq 1$. That $\left\|\phi_{1}\right\|=1$ will be noted later. 
Finally, putting $f=z$ in (1) we get, for $q \in Q,(u z)(q)=(u 1)(q) \phi(q)$. Thus $\phi=\phi_{1}$ on $Q$. This implies $\left\|\phi_{1}\right\|=1$. It also implies from (1) that, for $f \in A$, $u f=(u 1) f\left(\phi_{1}\right)$ holds on $Q$. Again since these functions are in $H^{\infty}, u f=(u 1) f\left(\phi_{1}\right)$ holds on $D$, so $u$ is a Type 1 operator.

This finishes the case where $m(Q)>0$. Now assume $m(Q)=0$, and we will show that $u$ is a Type 2 isometry. This falls immediately out of Proposition 3 . Let $Q, \rho: Q \rightarrow T$ and $\phi: Q \rightarrow T$ be as given in Proposition 3. Then

$$
\rho(q) u(f)(q)=f(\phi(q)) \text { for } f \in A \text { and } q \in Q .
$$

Let $\psi=\rho^{-1}$. We must show that $u f=E(\psi \cdot f(\phi))$, where $E: Y \rightarrow A$ is an extension operator on $Y=\{\psi \cdot f(\phi): f \in A\}$. We simply define the operator $E$ on $Y$ by $E(\psi \cdot f(\phi))=u f$. Then (4) shows that $E$ is an extension operator, and this finishes the proof of Theorem A.

Proof OF TheOrem B. The construction of $Q \subset T, \phi: Q \rightarrow K$, and $\psi \in$ $C(Q)$ is the same argument as Proposition 3. It only remains to show that $m(Q)=$ 0 . We suppose $m(Q)>0$ and get a contradiction. First we show that if $F$ is a closed subset of $Q$ with $m(F)>0$ then $\phi(F)=K$. For if $k_{0} \in K$ and $k_{0} \notin \phi(F)$, we can choose $f \in C(K)$ such that $f=1$ on $\phi(F)$ and $f\left(k_{0}\right)=0$. Then $u 1$ and $u f$ are identical on $F$, which is impossible since $m(F)>0$.

Now fix $k_{0} \in K$. Then $m\left(\phi^{-1}\left(k_{0}\right)\right)=0$. So there is an open subset $U$ of $T$ with $\phi^{-1}\left(k_{0}\right) \subset U$ and $m(U)<m(Q) / 2$. But, letting $F=Q \backslash U, m(F)>0$, and $\phi(F) \neq K$ since $k_{0} \notin \phi(F)$. This contradiction shows that $m(Q)=0$ and Theorem $\mathrm{B}$ is proved.

3. Some further remarks. We remarked earlier that one reason for additional isometries on $A$, besides the natural Type 1 isometries, is that the disc algebra contains subspaces isometric to $C(T)$ which naturally contains $A$. However, not all Type 2 isometries are restrictions to $A$ of isometries of $C(T)$, as illustrated by the following example (which appears in Rochberg [31]).

EXAMPLE. Let $Q$ be a closed subset of $T$ which is homeomorphic to the Cantor set and has Lebesgue measure 0. Let $\phi$ be a continuous map of $Q$ onto $T$. By Rudin's Theorem [25] there are functions $\phi_{1}$ and $\phi_{2}$ in $A$ such that $\phi_{1}=\phi_{2}=\phi$ on $Q$, but $\phi_{1} \neq \phi_{2}$ and $\left\|\phi_{1}\right\|=\left\|\phi_{2}\right\|=1$. Now define $u: A \rightarrow A$ by $u f=$ $\frac{1}{2}\left(f \circ \phi_{1}+f \circ \phi_{2}\right)$.

It is not difficult to check that $u$ is an isometry which is not Type 1 , so it must be Type 2. But there can be no isometry $w: C(T) \rightarrow A$ such that $w=u$ on $A$, since then $w(1)=u(1)=1$, and this is impossible (the argument here is exactly that used in the proof of Example 9.1 in Michael and Pelczynski [18]).

We close with a related problem about isometries which seems to be open.

Problem. Describe the isometries of $H^{\infty}$ into itself.

\section{REFERENCES}

1. D. E. Aslpach, Small into isomorphisms on $L_{p}$ spaces, Illinois J. Math. 27 (1983), 300-314.

2. J. Arazy, The isometries of $C_{p}$, Israel J. Math. 22 (1975), 247-256.

3. Y. Benyamini, Small into isomornhisms between spaces of continuous functions, Proc. Amer. Math. Soc. 83 (1981), 479-485.

4. E. Bishop, A general Rudin-Carleson theorem, Proc. Amer. Math. Soc. 25 (1958), 283-290.

5. M. Cambern, Isometries of certain Banach algebras, Studia Math. 25 (1965), 217-225. 
6. L. Carleson, Representations of continuous functions, Math. Z. 66 (1957), 447-451.

7. N. Dunford and J. T. Schwartz, Linear operators. I, Interscience, New York, 1958.

8. J. A. Erdos, A simple proof of Arazy's theorem, unpublished manuscript.

9. F. Forelli, The isometries of $H^{p}$, Canad. J. Math. 16 (1964), 721-728.

10. _ $\ldots$ theorem on isometries and the application of it to the isometries of $H^{p}(S)$ for $2<p<\infty$, Canad. J. Math. 25 (1973), 284-289.

11. K. Hoffman, Banach spaces of analytic functions, Prentice-Hall, Engelwood Cliffs, N.J., 1962.

12. W. Holsztynski, Continuous mappings induced by isometries of spaces of continuous functions, Studia Math. 26 (1966), 133-136.

13. C. Kolaski, Isometries of Bergman spaces on bounded Runge domains, Canad. J. Math. 33 (1981), 1157-1164.

14. __ Isometries of weighted Bergman spaces, Canad. J. Math. 33 (1981), 1156-1163.

15. A. Koranyi and S. Vagi, Isometries of $H^{p}$ spaces of bounded symmetric domains, Canad. J. Math. 28 (1976), 334-340.

16. J. Lamperti, On the isometries of certain function spaces, Pacific J. Math. 8 (1958), 459-466.

17. K. de Leeuw, W. Rudin and J. Wermer, The isometries of some function spaces, Proc. Amer. Math. Soc. 11 (1960), 694-698.

18. E. Michael and A. Pelczynski, A linear extension theorem, Illinois J. Math. 11 (1967), 563-579.

19. V. D. Pathak, Isometries of $C^{(n)}[0,1]$, Pacific J. Math. 94 (1981), 211-222.

20. _ Linear isometries of spaces of absolutely continuous functions, Canad. J. Math. 34 (1982), 298-306.

21. A. Pelczynski, On simultaneous extension of continuous functions, Studia Math. 24 (1964), 286304.

22. __ Addendum to: On simultaneous extensions of continuous functions, Studia Math. 25 (1964), 157-161.

23. _ - On $C(S)$-subspaces of separable Banach spaces, Studia Math. 31 (1968), 513-522.

24. N. V. Rao and A. K. Roy, Linear isometries of some function spaces, Pacific J. Math. 38 (1971), 177-192.

25. W. Rudin, Boundary values of continuous analytic functions, Proc. Amer. Math. Soc. 7 (1956), 808-811.

26. __ Real and complex analysis, McGraw-Hill, New York, 1966.

27. __ , $L^{p}$-isometries and equimeasurability, Indiana J. Math. 25 (1976), 215-228.

28. R. Schneider, Isometries of $H^{p}(U)$, Canad. J. Math. 25 (1973), 92-95.

29. K. W. Tam, Isometries of certain function spaces, Pacific J. Math. 31 (1969), 233-246.

30. F. T. Birtel, editor, Function algebras (Proc. Internat. Sympos. Function Algebras, Tulane Univ., 1965), Scott, Foresman, Glenview, Ill., 1966.

31. R. Rochberg, Which linear maps of the disc algebra are multiplicative?, Pacific J. Math. 38 (1971), 207-212.

32. J. N. McDonald, Isometries of function algebras, Illinois J. Math. 17 (1973), 579-583.

33. __ Isometries of the disc algebra, Pacific J. Math. 58 (1975), 143-154.

Department of Mathematics, OKlahoma State University, Stillwater, OKLAHOMA 74076

Department of Mathematics and Computer Science, Kansas Newman ColLEGE, WICHITA, KANSAS 67213 